as particles, have wave properties. Quantum theory confines itself to predicting the probabilities of different outcomes of experimental measurements (usually of particle properties), which can be calculated using the wavefunction.

Schwinger's philosophical approach would be considered pretty conventional today. He describes and approves of Bohr's principle of complementarity, which claims that certain observations can never be made simultaneously - for example, an electron can never be seen as a particle and a wave at the same time. And, in common with many of his contemporaries, he shows little awareness of the fundamental 'measurement problem' in quantum mechanics, which Schrödinger had exposed with the help of his famous cat and which many believe remains unresolved to this day.

To determine a set of mathematical tools for describing quantum mechanics, Schwinger analysed many variants of the Stern-Gerlach experiment. This experiment, which involves shooting a beam of atoms through a magnetic field, measures the quantized angular momentum of an atom. Schwinger's results enabled him to establish the now-familiar algebra of operators and state vectors. This approach contrasts with the more traditional one, which starts with the Schrödinger equation and the wavefunction for a free particle. Yet in many ways it parallels the treatment in Feynman's red book, although it is far more mathematical and formal. It is therefore surprising to come across an explicit derivation of an elementary trigonometric identity. Did Schwinger anticipate the lack of fluency in basic mathematics that is so common among modern students?

The scope of the later sections of the book is extensive, though not exhaustive. The section entitled 'Quantum dynamics' treats the standard problems of the harmonic oscillator in one, two and three dimensions. And the case where the oscillator is driven by a time-varying force is also included. The hydrogen atom is discussed, using an interesting analogy with the two-dimensional oscillator problem. In a section of the book that covers 'Interacting particles', we find an exhaustive discussion of the two-particle Coulomb problem and a chapter on identical particles that introduces quantum field theory. The latter topic is developed further in a final chapter on electromagnetic radiation.

So, do we have the "perfect way to teach quantum mechanics"? Probably not, if only because perfection is generally unattainable. What we do have is an interesting example of the way the subject was understood by one of the greatest physicists of the last century.

Alastair I. M. Rae is in the School of Physics and

Astronomy, University of Birmingham,

Birmingham B15 2TT, UK.

\section{Science in culture}

\section{Mellifluous mathematics}

Helaman Ferguson's topological sculpture. Martin Kemp

There are artists who engage effectively with science. And there are scientists involved at a high level in various arts. But there are few who can proffer such a unified curriculum vitae in art and science as the mathematical sculptor and sculptural mathematician, Helaman Ferguson.

Originally apprenticed to his stonemason adopted father, Ferguson studied painting, sculpture and mathematics at Hamilton College in New York, and subsequently gained a doctorate in mathematics from the University of Washington in Seattle. He taught mathematics for 17 years at Brigham Young University in Utah and was co-discoverer in the late 1970s of the first generalized euclidean algorithm. His sculptures are founded on advanced topological theorems of plastic geometry, working variations on forms with such picturesque names as Alexander's

horned wild spheres, toruses with cross-caps and Thurston's hyperbolic knotted wye.

Such a literally calculated approach might lead us to expect that Ferguson's sculptures would be cool abstractions in hard stone or intellectual conundrums in gleaming metal. Yet, in reality, they are beguilingly sensual and organic, subtly responsive to light and the mobile viewer, stimulating our tactile instincts in a remarkably seductive manner.

A characteristic example, on show at the National Science Foundation in Arlington, is Whaledream II, carved from a wonderful piece of Carrara marble. It assumes the form of a horned wild sphere in which the branched outer contour of the sphere's surface actively encompasses intricate spaces (in contrast to a standard sphere in which the inside of the surface simply surrounds a spherical space in the normal way). When we look at the complex mathematical form arising from this intellectual bedrock, we are presented not with a dry demonstration but with a mellifluous series of embracing curves that speak serenely of physical and emotional harmony. Cool calculation generates warm feelings. How can we explain the apparent paradox?

At one level, the reconciliation is affected by Ferguson's sensual intuition as a carver and skilled modeller, a master craftsman who delights in the inherent responsiveness of different materials, alternately resistant to stretching and compression. At the morphological level, however, there is no paradox at all. Ferguson has shown how topology stands alongside other geometries that generate forms analogous to those seen in the natural world. Alongside D'Arcy Thompson's more euclidean formulae of growth and form, and, more recently, alongside the new mathematics of complexity, chaos theory, fractals and self-organized criticality, the repertoire of shapes is aligned generically with such natural

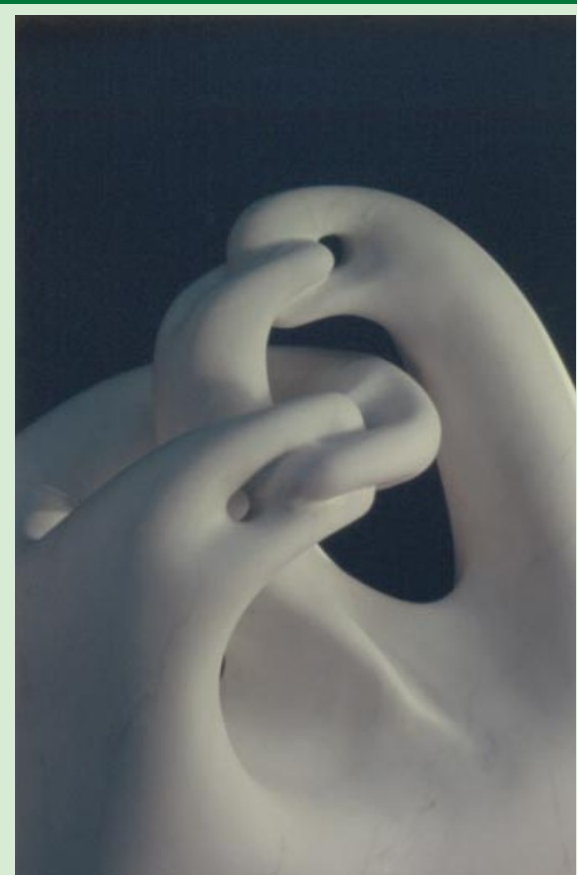

A horned wild sphere: Ferguson's Whaledream II.

structures as shells, horns, tendrils and softbodied marine organisms. He is inventing a form of living engineering that exists in parallel to the organic architecture of nature.

Ferguson recalls a recent demonstration of the probity of his structural principles. He points to a photograph in the National Science Foundation exhibition. It depicts "a negative gaussian curvature carving I did in 20 tons of snow at the ski resort in Breckenridge, Colorado [for an international snow sculpture competition]. I carved the snow walls down to about 4 inches thick. The next week Breckenridge had a heat wave and all the other sculptors' works sagged and imploded. Mine just got thinner and more gracile, retaining its form. The reason seems to be that on a negative gaussian curvature carving every point is a saddle point, every point is a keystone of a fabric of arches. The snow event was a proof of a simple but beautiful mathematical principle."

It is clear that we react intuitively at a very deep level to the morphological 'rightness' that is eloquently embodied in forms that are at once invitingly complex and elegant. Ferguson's creations work compellingly with our fundamental human instinct for the special kinds of mathematical order that are embedded in the world we see around us.

Martin Kemp is in the Department of the History of Art, University of Oxford, 59 George Street, Oxford OX1 2BE, UK.

Helaman Ferguson's sculptures and photographs are on view in "The Statue in the Stone" exhibition at the National Science Foundation, Arlington, Virginia, until 31 July 2001. 